\title{
Personalized Geolocation Image Tagging On Social Media
}

\author{
Abhjit V. Mophare \\ NBNSCOE, Solapur \\ Solapur University, Solapur
}

\author{
Anuradha S. Lamkane \\ NBNSCOE, Solapur \\ Solapur University, Solapur
}

\begin{abstract}
Social media has become prominent part of today's youth life. Social media has provided a space to share thoughts, share knowledge. Even if more of social media has been discussed for its negative impact on today's youth life, social media plays important role in organizing / gathering a community depends on relations, thoughts, education, work and religion. A photo tagging has made important role in organizing community. Tag is referred as word used by users to define or describe information in lighter way. Social tagging of personalized photo is to publically share photo o social sites. In this paper personalized tags recommendation task are focused and userpreferences, geo-location-specific tags are identified to relate community. A large number of users and geo location specific photos are used for experimental purpose. The user based tags can be used to describe community and photos can be used evaluate popularity of specific location.
\end{abstract}

\section{Keywords}

Geo-location preference, personalized tag recommendation, subspace learning, tagging history, user preference.

\section{INTRODUCTION}

$21^{\text {st }}$ Century is known as communication century and Internet has become heart of this communication. There are approximate 4 billion internet users [2] and 2.23 billion active social networking users [3] in world. Social media allow people to communicate and share text, images and videos. Social media is also positively involved in building and strengthen relation. Social media websites like Facebook, Twitter, LinkedIn, Google+, instagram etc allowed user to share multimedia.

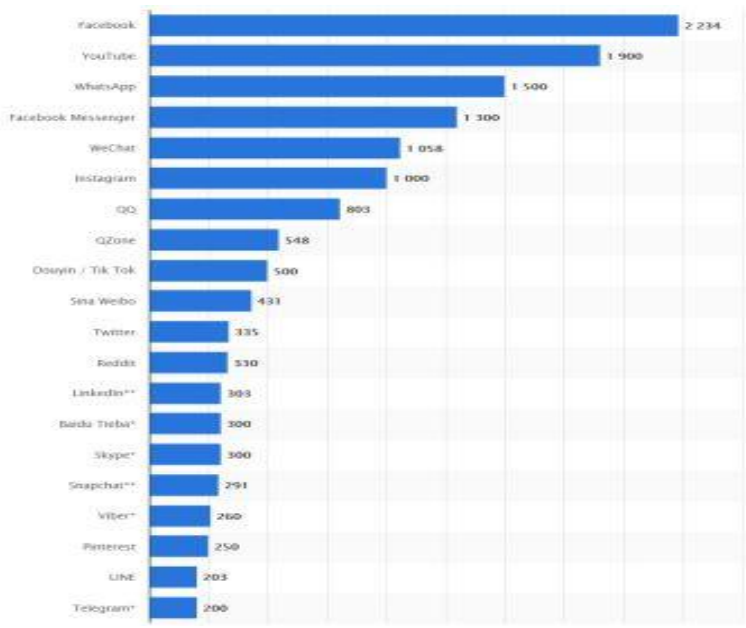

Fig 1: Number of active social media users

The photo sensing devices are normally equipped with GPS mechanism, people used to take photos and tag with location and description. Social media now days allows user to share images with proper security and privacy. As a consequence, photos tagged by community and friends on social media can consume space from social media or personal devices. As photos are uploaded, definitely efficient tag recommendation, image retrieval and annotation mechanism is required [4]. Challenges of building community and tagging are irrelevant tags and unknown photo tagging. The solution to this is explained in [5], [6], [7], [8], [9]. Tagged photos should be clear, uniform sized and proper tags should be used for annotations.

If a user is unaware of standard recommendation mechanism, user can tag different image for same geo-location or user can use different tags for same geo-location with different images.

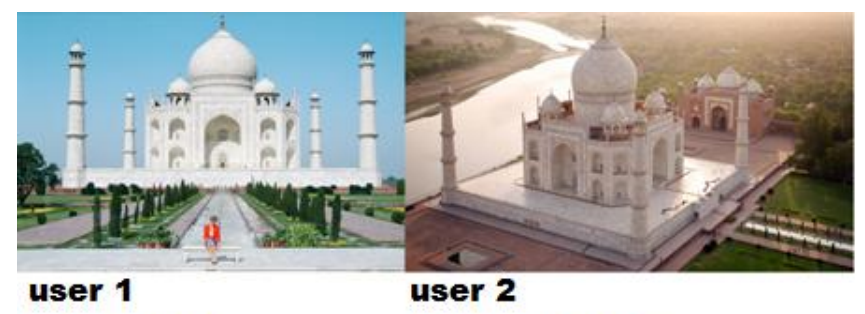

tag: Taj mahal

tag : Taj 7th wonder

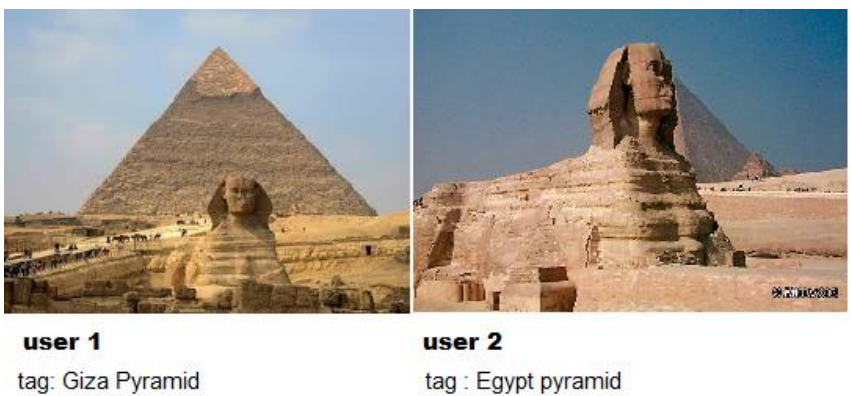

(a) Taj mahal images with different Users and different

(b) Giza pyramid images with different tags

tags

Fig. 2 Tag recommendation of users and geo-locations

Investigating geographic preferences or popularity index towards tags from huge amount of context multimedia is challenging task. To address this issue a personalized geolocation tags framework is designed. The framework designed contains geo-location attribute like longitude and latitude specific images. Different users on social media allowed searching specific photos and tagging photos.

The paper is organized like I part contains introduction II contains related work, III part contains design and implementation and result discussion while last part IV concludes current work. 


\section{RELATED WORK}

Tagging is mechanism where a user defined words are attached with image, video etc multimedia data, which will be used by the reader /observer for better understanding of multimedia data.

Yang Song, Lu Zhang and C. Lee Giles have proposed two novel centered approaches that are capable of making efficient tag attachment with real scenarios [10]. The graph based method and prototype based method, are emphasizing on bipartite graph and a sparse multiclass Gaussian process classifier for efficient document classification. The method proved as efficient tagging mechanism but mechanism is complex and expensive.

N. Garg and I. Weber presented a personalized approach to tag recommendation for Flickr photos [11]. They presented comparison of three methods a) query-independent personal tag usage b) query dependent personal tag usage c) query dependent group tag depending on the group the photo. It does not contain a user social context approach. They highlight the good performance of a hybrid method combining the personal and general contexts that gives improvement over either context alone. They have demonstrated balancing between personal and general evidence. When personal tag recommendation and group recommendation are combined, users who tag a lot tend to benefit relatively less from general evidence and more from personal evidence.

Rashmi Sinha has considered tagging from the perspective of awareness [12]. Rashmi Sinha stated that taggers used to use free words to describe social environment. The tag and image can be viewed by all users and can give feedback on geo location and image. The emerged group can be used to share their thoughts and experience. The emerged group can become a forum for discussion. Tagging can act as bridge between personal and social environment. The most popular tag can be calculated where meaning to that image / geo-location can be evaluated. The analysis was used to prove most meaningful image is the one which was tagged by most of users on social media websites.

Jing Li, Xueming Qian proposed a hierarchical structure to calculate GPS location of an image [13]. Hierarchical structure is cost effective for GPS estimation performance and computational cost. A large set of tags are divided into small groups or clusters. The cluster contains representatives of images rather than actual images. Hence the local features are mapped with image representative rather than actual image. The mechanism is cost effective.

Huagang Yin, Changhu Wang developed a path search system to promote tourists' trip planning [14]. They have developed a mechanism which will guide tourist not only where to visit but also suggest how to visit. They have demonstrated mechanism over more than 20 million photos. The photos are geo tagged and suggest multiple routes to tourist. The experimental results proved to be rich paths from source to destination. The calculated path doesn't have any relation with shortest path from source to destination. It acts as smart guide which is used to ease travel plan of tourists.

In this paper [15], we have presented a novel automatic trip planning framework, by leveraging Web scale geo-tagged photos and textual travelogues. Owning to the 20 million geotagged photos, an online system has been developed to help users plan travel routes for over 30; 000 landmarks in more than 100 countries and territories. To the best of our knowledge, it is the first research work to systematically investigate the trip planning problem. It is also the first system that could interactively help users plan travel routes. Experimental results have shown the intelligence and effectiveness of the proposed framework.

An-Jung Cheng, Yan-Ying Chen, have proposed a probabilistic personalized travel recommendation model which adopts the automatically mined knowledge from the travel photo logs and the automatically detected people attributes in the photo contents [16]. By information-theoretic measures and experiments in eight major cities, people attributes are effective for mining demographics for travel landmarks and paths and very helpful for personalized travel recommendation and route planning. People attributes are orthogonal to the travel logs alone and can further yield more satisfactory results especially in more challenging recommendations. They conducted experiments on more people attributes and adjust the probabilistic model for such diverse attributes to address more capabilities in personalization. Besides, the more competitive recommender models need to be investigated as well. They have experimented on four million photos collected for eight major worldwide cities. They have introduced probabilistic Bayesian learning framework which further entails mobile recommendation on the spot.

Roelof van Zwol, Borkur Sigubjornsson suggested a tag based on tag repository of all users reside in Flickr [17]. Given a photo with tags, $m$ number of ordered candidate tags are derived for each of the user-defined tags, based on tag co-occurrence. These tags are used as input for aggregation and ranking, which produces $\mathrm{n}$ number of ranked tags to be recommended. The recommended tags are used as an index for an image retrieval system. The experiment is conducted on 331 images downloaded from Flickr. The proposed method is good for recommending locations, objects tags. But it is computationally expensive to work on all images and tags.

Nikhil Garg proposed work to suggest a tag by exploring user's previous tagging history [18]. When a user assigns tags for an image, the system suggests tags based on the tags that have used in user past along with the tags already entered. The user may select tags from suggested list or simply ignore it and add an own tag to an image. The Hybrid algorithm combining conservative performance, classical classification and new cost measure is used in the proposed work. The advantage of proposed work is the low average cost of the tagging. The work did not explain about the goodness of recommended tag.

Aixin Sun developed the knowledge-based approach for image tag recommendation which exploits tag concepts [19]. Tag concepts are derived based on tags of images represented in the form of tag co-occurrence pairs. The tag relationship graph is constructed for each candidate tag. Then modularity clustering is used to identify the concept associated with each tag. These concepts are used as an index. The matching concepts are retrieved from the index using cosine similarity. Finally, the candidate tags associated with a matching candidate is recommended. The method boosts scalability and efficiency of tag recommendation process. However, it does not consider features of the images to improve visual search.

Luca Cagliero [20] developed a method to suggest tags based on generalized association rules which explore tags correlation holding at different abstraction levels to identify additional tags for a suggestion. The method store tag set in a transactional data and a generalization hierarchy GH is built over transaction tag. Given the minimum threshold and support, GENIO algorithm is used over GH to generate user specific rule set and collective rule set. Using these rules, tags are selected and are ranked using Borda Count Consensus function. Due to the generalized rule set, the performance of the recommender system is very high. 
But the developed system does not work well for the images with noisy tags.

Adam Rae developed tag recommendation system to suggest the additional tag for partially annotated images [21]. The recommended system recommend tags using utilizing the knowledge that present at four different contextual layers using the probabilistic framework. The first contextual layer is personal context, constructed from the annotations provided by the user. Secondly, a social contact context is defined by aggregating the annotations over all users that are identified as a contact of that user. Thirdly, a social group context is obtained by aggregating the photo annotations of photos posted in the groups that the user is subscribed to. Finally, a collective context is determined by aggregating the annotations for all photos posted by all users. The tags are extracted for each context based on repetitive occurrence analysis of tags used to annotate the photos within that context. To produce a set of recommendations for a given set of input query tags, each query tag is used to generate an intermediate set of recommendations and these sets are then combined. For each of the contexts, the same probabilistic model and Borda Count based aggregation approach are used to generate recommendations from different contexts into a unified ranking of recommended tags. The method is able to recommend relevant tags to an image irrespective of language.

Xian Chen and Hyoseop Shin developed an algorithm to suggest tag using both textual and social features [22]. Through both textual analysis and social activities, the algorithms collect features for tags of each user. To find tags which are related to user's interest textual features are extracted from each user's own terms in tags, titles, contents, and comments which are applied to his/her own photos. Also, the user favorite topic is determined from some tags used frequently. Social features are determined from each user's social activities e.g. a user marks other users' photos as his/her favorites, or another user marks his/her own photos as favorites. If users marked other several photos which are related to the same tags, the algorithm infers that users are interested in the topics on these tags. Naive Bayes classifier is used to find the representative tags that can be related to the users' favorite topics. The algorithm is good at finding highquality tags for items. The advantage of the technique is: it is easy to compute and previously assigned tags are easily available. But these tags are highly personalized and may not be relevant to images. The solution is to use image features along with tags for accurate tag recommendation.

\section{OVERVIEW OF OUR SOLUTION}

The intention of the personalized geo-location tagging is to build community based on liking of uploaded images on social media. It provides a forum for communication.

There are two types of users
i) Owner
ii) user

Owners have the authority to upload images with latitude and longitude with description.

Users can have following authorities

Search Users can search all images uploaded by all owners of images.

Tag: Tag is user defined word to describe a place or geolocation. Users / Owners can tag the photo; in other words users and owners have freedom to express their views about location or image in their own word. Depending on number of tags most popular images can be evaluated.
It is an offline process where the work can be defined as collection of following processes

Image collection: A large number of photos are collected to upload on social media. Mainly the image belongs to famous places, temples, churches and natural places. It supports all types of images for uploading includes bmp, png, jpg, jpeg etc...
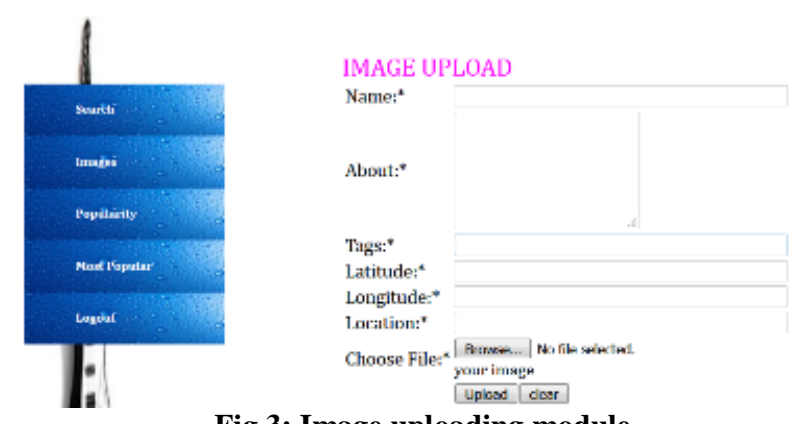

Fig 3: Image uploading module

Name of image, description of image / place, tag, latitude , longitude and image can be uploaded. The image can be saved in mysql database as longblob data type.

Search: User can search the images depending on name of image or tag name. The image can be retrieved with image information, latitude, and longitude with two options.

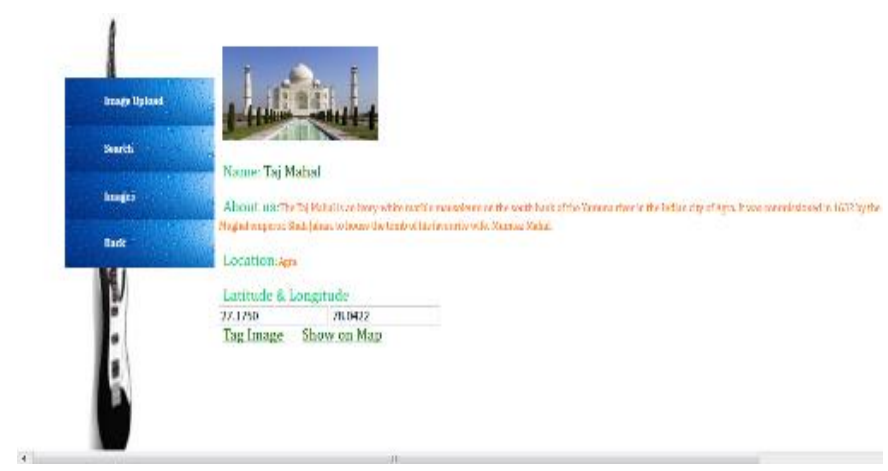

Fig 4: Tagging module

\section{Option1: Tag Image}

User can tag image. The tag can be appended with previous tags. If a image is associated with multiple tags; any of tag can be used to search image.

Option 2: Show on map

The project can take input as latitude and longitude and used to display the location on map.

\section{Popularity:}

An image can be decided as popular depending on number tags associated with image. The image associated with maximum tag can be considered as most popular image of database. User / Owner can view popularity of images and most popular image of offline image database. 


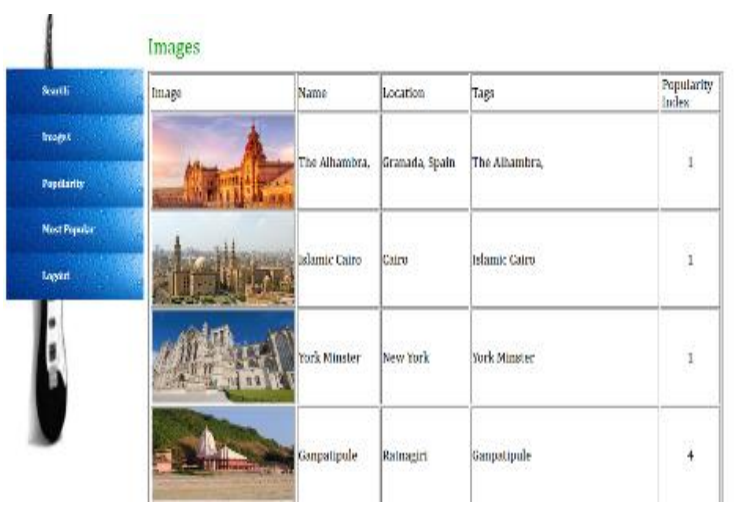

(a)

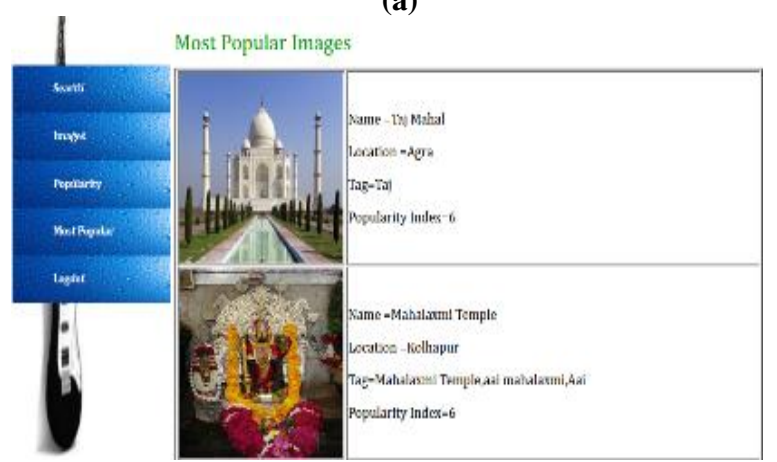

(b)

Fig 5: Popularity index and most popular images module

\section{IMPLEMENTATION}

The mechanism is implemented in windows environment with JSP, Servlet with MySQL as database. Approximately 1300 images have been uploaded on social media. The different types of images, with different dimensions and sizes can be uploaded.

400 users of type owner and normal users are created. Owners have authority to define image while normal users can search, tag and see popularity of images.

Google map API are used to show image on map. To show particular location on map, module can take input as latitude and longitude.

The result of tag recommendation can be viewed as follows.

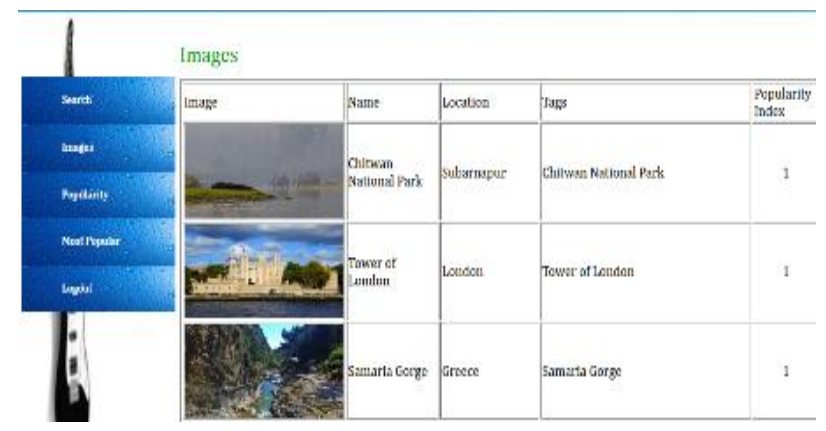

Fig 6: Popular index module

The result contains image, name, location, tags and popularity index.

\section{CONCLUSION}

The personalized geo-location tagging can be used to create community or group depending on tagging of images. It provides features of searching image, popular image and most popular image of uploaded image. Many recommendation methods have been developed used to recommend relevant tag for image but tag image association is not sufficient. Our approach provides a free space to tag image with user defined image. Further as a future work, an image uploaded namely called as owner can act as admin for group of users who have tagged image over social media site.

\section{REFERENCES}

[1] Jing Liu, Zechao Li, Jinhui Tang, Yu Jiang, "Personalized Geo-Specific Tag Recommendation for Photos on Social Websites", IEEE TRANSACTIONS ON MULTIMEDIA, VOL. 16, NO. 3, APRIL 2014

[2] https://wearesocial.com/blog/2018/01/global-digital-report2018

[3] https://www.statista.com/statistics/272014/globalsocialnetworks-ranked-by-number-of-users/

[4] T. Mei, W. H. Hsu, and J. Luo, "Knowledge discovery from community contributed multimedia," IEEE Multimedia, vol. 17 , no. 4, pp.16-7, Oct. 2010.

[5] Y. Shen and J. Fan, "Leveraging loosely-tagged images and inter-object correlations for tag recommendation," in Proc. ACM Multimedia, 2010.

[6] J. Tang, S. Yan, R. Hong, G.-J. Qi, and T.-S. Chua, "Inferring semanticconcepts from community-contributed images and noisy tags," in Proc. ACM Multimedia, 2009.

[7] H. Chen, M. Chang, P. Chang, M. Tien,W. Hsu, and J.Wu, "Sheepdog: Group and tag recommendation for flikr photos by automatic searchbased learning," in Proc. ACM Multimedia, 2008

[8] Y. Song, L. Zhang, and C. L. Giles, "Automatic tag recommendation algorithms for social recommender systems," ACM Trans. Web, vol. 5,no.1, 2011.

[9] A. Sun, S. S. Bhowmick, and J.-A. Chong, "Social image tag recommendation by concept matching," in Proc. ACM Multimedia, 2011.

[10] Y. Song, L. Zhang, and C. L. Giles, "Automatic tag recommendation algorithms for social recommender systems," ACM Trans. Web, vol. 5,no. 1, 2011.

[11] N. Garg and I. Weber. Personalized, interactive tag recommendation for ickr. In ACM Conference on Recommender Systems, 2008, pages 67\{74.

[12] Sinha, R. (2005). A social analysis of tagging. Available at: http://blog.jackvinson.com/archives/2005/10/01/a_cognitiv e_analysis_of_tagging.html (accessed 29 Sep 2008).

[13] J. Li, X. Qian, Y. Y. Tang, L. Yang, and T. Mei, "Gps estimation for places of interest from social users' uploaded photos," IEEE Transactions on Multimedia, vol. 15, no. 8 , pp. 2058-2071, 2013.

[14] H. Yin, C. Wang, N. Yu, and L. Zhang, "Trip mining and recommendation from geo-tagged photos," in IEEE International Conference on Multimedia and Expo Workshops. IEEE, 2012, pp. 540-545.

[15] Xin Lu, Changhu Wang, Jiang-Ming Yang, Yanwei Pang, and Lei Zhang. Photo2Trip: Generating Travel Routes from Geo-Tagged Photos for Trip Planning. ACM Multimedia 2010

[16] An-Jung Cheng, Yan-Ying Chen, Yen-Ta Huang, Winston H. Hsu, and Hong-Yuan Mark Liao. Personalized travel recommendation by mining people attributes from 
community-contributed photos. ACM Multimedia 2011

[17] B. Sigurbjonsson and R. V. Zwol, "Flickr tag recommendation based on collective knowledge," in Proc. ACM International Conference on World Wide Web, 2008, pp. 327-336.

[18] N. Garg and I. Weber, "Personalized interactive tag recommendation for Flickr," in Proc. ACM Conference on Recommender Systems, 2008, pp. 67-74.

[19] A. Sun, S. S. Bhowmick, and J. A. Chong, "Social image tag recommendation by concept matching," in Proc. ACM International Conference on Multimedia, 2011, pp. 11811184.
[20] L. Cagliero and P. D. Torino, "Personalized tag recommendation based on generalized rules," ACM Transactions on Intelligent Systems and Technology, vol. 5, no. 1, pp. 12:1-12:22, 2013.

[21] A. Rae, B. Sigurbjrnsson, and R. V. Zwol, "Improving tag recommendation using social networks," in Proc. Adaptivity, Personalization and Fusion of Heterogeneous Information, 2010, pp. 92-99.

[22] X. Chen and H. Shin, "Tag recommendation by machine learning with textual and social features," Journal of Intelligent Information Systems, vol. 40, no. 2, pp. 261-282, 2013. 